\section{(6) OPEN ACCESS}

\title{
Erosive osteoarthritis: a more severe form of radiographic hand osteoarthritis rather than a distinct entity?
}

\author{
Michelle Marshall, ${ }^{1}$ Elaine Nicholls, ${ }^{1}$ Wing-Yee Kwok, ${ }^{2}$ George Peat, ${ }^{1}$ \\ Margreet Kloppenburg, ${ }^{2}$ Danielle van der Windt, ${ }^{1}$ Helen Myers, ${ }^{1}$ Krysia Dziedzic ${ }^{1}$
}

\begin{abstract}
Handling editor Tore K Kvien
- Additional material is published online only. To view please visit the journal online (http://dx.doi.org/10.1136/ annrheumdis-2013-203948).

${ }^{1}$ Arthritis Research UK Primary Care Centre, Primary Care Sciences, Keele University, Staffordshire, UK

${ }^{2}$ Department of Rheumatology, Leiden University Medical

Center, Leiden,

The Netherlands
\end{abstract}

\section{Correspondence to}

Dr Michelle Marshall, Arthritis Research UK Primary Care Centre, Primary Care Sciences, Keele University, Staffordshire ST5 5BG, UK; m.marshall@ keele.ac.uk

Received 14 May 2013 Revised 9 August 2013 Accepted 20 September 2013 Published Online First 4 October 2013

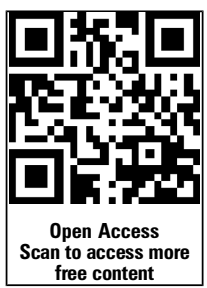

\section{CrossMark}

To cite: Marshall $M$, Nicholls E, Kwok W-Y, et al. Ann Rheum Dis

2015;74:136-141.
ABSTRACT

Objectives To determine whether erosive osteoarthritis shares the same pattern of joint involvement and risk profile as increasing grades of non-erosive hand osteoarthritis.

Methods Participants were from two population-based cohorts, aged $\geq 50$ years, reporting hand symptoms in the previous month. Interphalangeal joints were assessed for erosive osteoarthritis (Verbruggen-Veys erosive or remodelled phase) and radiographic osteoarthritis (sliding cut-offs of $K \& L \geq 2, K \& L \geq 3$ and $K \& L=4$ ). At the joint level, similarities in the frequency and pattern of erosive and nonerosive osteoarthritis were assessed by Spearman's rank correlation coefficients and generalised estimating equations. At the person level, individuals with erosive osteoarthritis were compared to those with non-erosive osteoarthritis using logistic regression, adjusted for age and gender $(\mathrm{aOR})$, for the following exposures: family history, previous injury, overuse and metabolic factors (BMI, dyslipidaemia, hypertension, diabetes).

Results In 1076 symptomatic participants the ranked frequency of involvement for erosive joints was comparable to joints with $K \& L \geq 3$ and $K \& L=4$ ( $r>0.95)$. Patterns of joint involvement in erosive osteoarthritis were strongest for symmetry (aOR=6.5; $95 \% \mathrm{Cl} 3.0$ to 14.1$)$, followed by row $(2.0 ; 0.8$ to 5.0$)$ and ray $(0.3 ; 0.0$ to 2.5$)$, which was similar to joints with $K \& L \geq 3$ and $K \& L=4$. Individuals with erosive osteoarthritis $(n=80)$ had an increased risk of metabolic syndrome (2.7; 1.0 to 7.1$)$, notably dyslipidaemia (4.7; 2.1 to 10.6) compared with nonerosive osteoarthritis classed $K \& L \geq 3(n=193)$.

Conclusions The similar frequency of radiographic joint involvement and patterning in erosive osteoarthritis and more severe non-erosive osteoarthritis is consistent with prevalent erosive osteoarthritis being a severe form of hand osteoarthritis rather than a distinct entity. Metabolic exposures, dyslipidaemia in particular, may be implicated in erosive osteoarthritis.

\section{INTRODUCTION}

Inflammatory osteoarthritis was first described by Crain ${ }^{1}$ in 1961 and the term erosive osteoarthritis was coined 5 years later by Peter et al. ${ }^{2}$ Erosive osteoarthritis predominantly affects the interphalangeal joints (IPJ) and is characterised by abrupt onset with inflammation. Those affected experience tissue swelling, stiffness, erythema, and report considerable pain and functional limitation. ${ }^{2-4}$ Hand radiographs demonstrate a characteristic central erosion with collapse of the subchondral bone and a 'gull-wing' or 'saw-tooth' deformity. ${ }^{4} 5$ Complete loss or pseudo-widening of the joint space may be apparent. ${ }^{6}$ Over time, subsequent reparative alterations are frequently seen with a new, but irregular subchondral plate and a new joint space. ${ }^{6}$ In this remodelling phase osteolytic areas gradually recede and large osteophytes develop. ${ }^{7}$

The prevalence of erosive osteoarthritis in the general population has been estimated at $2.8 \%$ rising to $10.2 \%$ in those with symptomatic osteoarthritis. ${ }^{8}$ Erosive osteoarthritis has been found to have poorer clinical and radiographic outcomes than non-erosive hand osteoarthritis. $^{9-12}$ European League Against Rheumatism (EULAR) recommendations for the diagnosis of hand osteoarthritis have suggested that erosive osteoarthritis should be considered as a subset of hand osteoarthritis. ${ }^{13}$ However, the etiopathogenesis is not clear and poorer clinical outcomes may be the result of erosive osteoarthritis being a discrete disease entity rather than a more severe form of hand osteoarthritis.

Studies and case reports have suggested that erosive osteoarthritis affects distal interphalangeal (DIP) joints more frequently than the proximal interphalangeal (PIP) joints and that it is highly symmetrical. $^{3} 14 \quad 15$ These are the same patterns of involvement that are reported in non-erosive hand osteoarthritis. ${ }^{16} 17$ Risk factors that are known for hand osteoarthritis include female gender, older age, family history, obesity, previous hand injury and occupational and recreational activities. ${ }^{13}$ Some of these risk factors, including a female predominance, obesity and a hereditary aspect, have also been reported for erosive osteoarthritis. ${ }^{18}$ To date, no studies have directly compared joint involvement and risk factors in erosive osteoarthritis to increasing grades of non-erosive osteoarthritis.

This study aims to investigate whether erosive osteoarthritis is a discrete disease entity or at the severe end of the hand osteoarthritis continuum. We examined similarities in the frequency and patterns of joint involvement between erosive osteoarthritis and increasing grades of non-erosive radiographic hand osteoarthritis, and compared risk factor profiles between individuals with erosive osteoarthritis and non-erosive radiographic hand osteoarthritis.

\section{METHODS \\ Study population}

Participants were recruited from a prospective observational cohort study undertaken in North 
Staffordshire: the Clinical Assessment Study of the Hand (CASHA). All adults aged 50 years and older registered with two general practices were invited to participate at baseline in a two-stage self-report questionnaire. ${ }^{19}$ In the UK over $95 \%$ of people are registered with general practices, thus providing convenient general population sampling frames. ${ }^{20}$ Those with hand pain or hand problems in the past 12 months were invited to research clinics that included an interview, clinical assessment and radiographs.

Participants reported the frequency of hand pain, aching or stiffness in the past month (no days, few days, some days, most days or all days). Those who reported symptoms on a few days or more were deemed symptomatic and eligible for inclusion in the analyses. Participants were excluded if general practice or local rheumatology hospital medical records or a consultant musculoskeletal radiologist identified them as having inflammatory arthritis (rheumatoid arthritis, psoriatic arthritis). Those with no hand radiographs or missing radiographic data were also excluded.

The sample was enriched from an identically performed survey in a similar population to increase the number of individuals with erosive osteoarthritis included in the analysis. In the Clinical Assessment Study of the Knee (CASK) all adults aged 50 years and older registered with three general practices in North Staffordshire were invited to participate at baseline in the same two-stage self-report questionnaire. ${ }^{21}$ Individuals reporting knee pain were invited to attend research clinics and received an identical hand assessment and hand radiographs to those in the CASHA study. All individuals included in the analysis had hand pain on a few days or more in the previous month.

The North Staffordshire Local Research Ethics Committee approved both these studies (LREC project no. 1430). All participants provided written informed consent.

\section{Data collection}

\section{Radiographic assessment and scoring}

Posterior-anterior radiographs of the hands were taken with separate exposures for each hand according to a standardised protocol. $^{19}{ }^{21}$ The Kellgren and Lawrence (K\&L) grading system was used by two trained readers (CASHA: MM; CASK: JH) to grade osteoarthritis in 16 joints in each hand. ${ }^{22}$ Intrarater reliability for the presence of osteoarthritis $(K \& L \geq 2)$ in an individual joint was excellent (unweighted mean kappa $=0.92$ and 0.85 , mean percentage agreement $=98 \%$ and $98 \%$ for reader 1 and 2, respectively) and interrater reliability was moderate (unweighted mean kappa $=0.5$, mean percentage agreement $=90 \%$ ). The presence of erosive osteoarthritis in the IPJ (rays 2-5) was determined using the Verbruggen-Veys anatomical phase progression score by a single reader (WYK), intrarater reliability was excellent (unweighted mean kappa $=0.94$, mean percentage agreement $=98 \%){ }^{6}$ Knee radiographs were also taken and scored for the presence of osteoarthritis. ${ }^{1921}$

\section{Risk factors}

Demographic and socioeconomic data including gender, age, occupation and education were collected in the baseline survey along with self-reported previous hand injury and excessive use of hands in either employment or pastimes. At the research clinics, participants were asked whether their parents or siblings had arthritis, and height and weight were measured to calculate the body mass index (BMI).

A review of general practice medical records was undertaken for a 2-year period before clinic attendance for participants providing permission $(\mathrm{n}=1007,94 \%)$. General practice consultations in the UK are coded for medical diseases, symptoms and procedures (read codes) using a hierarchical method. The five participating practices were fully computerised and the completeness of their coding of consultations has been subject to regular quality reviews. ${ }^{23}{ }^{24}$ Participants with a diagnosis and consultations for hypertension, type 2 diabetes or impaired fasting glucose and dyslipidaemia or prescription of a lipid-regulating drug were identified. Metabolic syndrome was defined as the presence of three or more of the following: BMI $>30 \mathrm{~kg} / \mathrm{m}^{2}$, hypertension, dyslipidaemia and type $2 \mathrm{dia}-$ betes or impaired fasting glucose.

\section{Case definitions}

All individuals included in this analysis were symptomatic (pain, aching or stiffness on a few days or more in the past month). Analysis was completed on two levels: joint and person.

Erosive osteoarthritis in a joint was defined as having eroded ' $E$ ' or remodelled 'R' phase of the Verbruggen-Veys anatomical phase progression score. Non-erosive radiographic hand osteoarthritis in a joint was defined by sliding cut-offs of $\mathrm{K} \& \mathrm{~L}$ grades: $\mathrm{K} \& \mathrm{~L} \geq 2, \mathrm{~K} \& \mathrm{~L} \geq 3$ and $\mathrm{K} \& \mathrm{~L}=4$.

At the person level, individuals with erosive osteoarthritis were defined as having one or more IPJ with eroded ' $\mathrm{E}$ ' or remodelled ' $\mathrm{R}$ ' phase of the Verbruggen-Veys anatomical phase progression score. Reference groups consisted of individuals free of erosive osteoarthritis who had increasing grades of radiographic osteoarthritis: $\mathrm{K} \& \mathrm{~L} \geq 2, \mathrm{~K} \& \mathrm{~L} \geq 3$ and $\mathrm{K} \& \mathrm{~L}=4$ defined by the highest grade present in the IPJ.

\section{Statistical analysis}

The frequency of joint involvement was examined for erosive osteoarthritis and increasing grades of non-erosive radiographic osteoarthritis by ranking the IPJ in the order that they were most frequency affected, and Spearman rank correlation coefficients were used to compare the rank order. Analysis of the patterns of joint involvement by symmetry (same joint opposite hand), row (same row same hand) and ray (same ray same hand) were undertaken using three separate generalised estimating equation models to account for the lack of independence in the data for multiple hand joints in a single individual. Using symmetry as an example, the aim of the model was to test whether having osteoarthritis in a single joint increased the odds of having osteoarthritis in the same joint on the opposite hand. All generalised estimating equation models were fitted using an exchangeable correlation structure and robust SE. Model results are presented as OR adjusted for potential confounding by age, gender, hand joint, hand side and the number of affected IPJ.

Analysis was undertaken at the person level to compare the descriptive characteristics and risk factors of individuals with erosive osteoarthritis to reference groups with increasing grades of non-erosive radiographic osteoarthritis. The OR for a risk factor in those with erosive osteoarthritis compared to reference groups were calculated, and adjusted for age and gender.

Analyses were performed using IBM SPSS Statistics V.20 and STATA V.11.0. All tests were two tailed and a $p$ value of less than 0.05 was considered statistically significant.

\section{RESULTS}

\section{Study population}

In total, 15396 adults aged 50 years and over from five general practices in North Staffordshire were mailed a two-stage survey from which CASHA and CASK study participants were recruited (see supplementary figure 1, available online only, for full details of the recruitment process). In total, 1167 
participants reporting hand pain on a few days or more in the previous month attended the research clinics (CASHA $n=578$; CASK $n=589$ ). Even though only a small proportion of those surveyed attended research clinics the CASK sample has been shown to be representative of the symptomatic general population and similar analyses in CASHA resulted in the same conclusions. $^{25}$

Following exclusions for inflammatory arthritis $(n=44)$, no hand X-rays $(n=6)$ and missing X-ray data $(n=41), 1076$ participants were included in the analysis (CASHA $n=521$; CASK $n=555$ ), $60 \%$ women, mean age 64.8 years (SD 8.3, range 50-93).

\section{Frequency of joint involvement\#\#}

In total, 8608 hand joints were examined, with $K \& L \geq 2$ present in 1754 joints, $\mathrm{K} \& \mathrm{~L} \geq 3$ in 425 joints, $\mathrm{K} \& \mathrm{~L}=4$ in 112 joints and erosive osteoarthritis in 207 joints.

Erosive osteoarthritis had the same rank involvement for the DIP joints as moderate-severe $(K \& L \geq 3)$ and severe $(K \& L=4)$ osteoarthritis in the right hand and severe osteoarthritis $(K \& L=4)$ in the left hand (table 1$)$. In both hands statistically significant associations were seen for the rank order of joints with erosive osteoarthritis and moderate-severe osteoarthritis $(\mathrm{K} \& \mathrm{~L} \geq 3)$, and for erosive osteoarthritis and severe osteoarthritis $(\mathrm{K} \& \mathrm{~L}=4)$ (table 1 , Spearman $\mathrm{r} \geq 0.95)$. Fewer similarities were seen when the frequency of erosive joint involvement was compared to the presence of any radiographic osteoarthritis $(\mathrm{K} \& \mathrm{~L} \geq 2)$ (table 1$)$.

\section{Patterns of joint involvement}

Trends in the patterning of joint involvement were strongest by symmetry followed by row and then ray for erosive osteoarthritis and increasing grades of radiographic osteoarthritis, although in erosive joints statistically significant odds were only seen for symmetrical patterning (table 2). The strength of association for symmetry and ray was similar for erosive osteoarthritis compared with moderate-severe $(K \& L \geq 3)$ and severe hand osteoarthritis $(\mathrm{K} \& \mathrm{~L}=4)$ (table 2$)$.

\section{Demographic and radiographic characteristics}

In total, 80 participants were classed as having erosive osteoarthritis (mean number of erosive joints $=2.6 \quad(\mathrm{SD}=1.9)$, range $=1-9$ ). As similarities were seen between erosive osteoarthritis and moderate-severe and severe osteoarthritis in the joint patterning, analyses focused on the reference groups of 193 individuals with $K \& L \geq 3$ and the 68 who had $K \& L=4$. In individuals with erosive osteoarthritis the distribution of gender and age was comparable to those with moderate-severe $(\mathrm{K} \& \mathrm{~L} \geq 3)$ and severe $(\mathrm{K} \& \mathrm{~L}=4)$ osteoarthritis (table 3). Individuals with erosive osteoarthritis had thumb metacarpophalangeal and first carpometacarpal joint osteoarthritis slightly more often while symptomatic radiographic knee osteoarthritis was less frequent compared to those with more severe non-erosive osteoarthritis (table 3); however, the 95\% CI showed none of these differences to be statistically significant (data not shown).

\section{Risk factors}

After adjustment for potential confounders, individuals with erosive osteoarthritis had increased odds of having dyslipidaemia $(\mathrm{aOR}=4.7)$ and a classification of metabolic syndrome $(\mathrm{aOR}=2.7)$ when compared to individuals with non-erosive radiographic osteoarthritis $K \& L \geq 3$ (table 4 ). The same patterns were seen when erosive osteoarthritis was compared to the reference group with $\mathrm{K} \& \mathrm{~L}=4$.

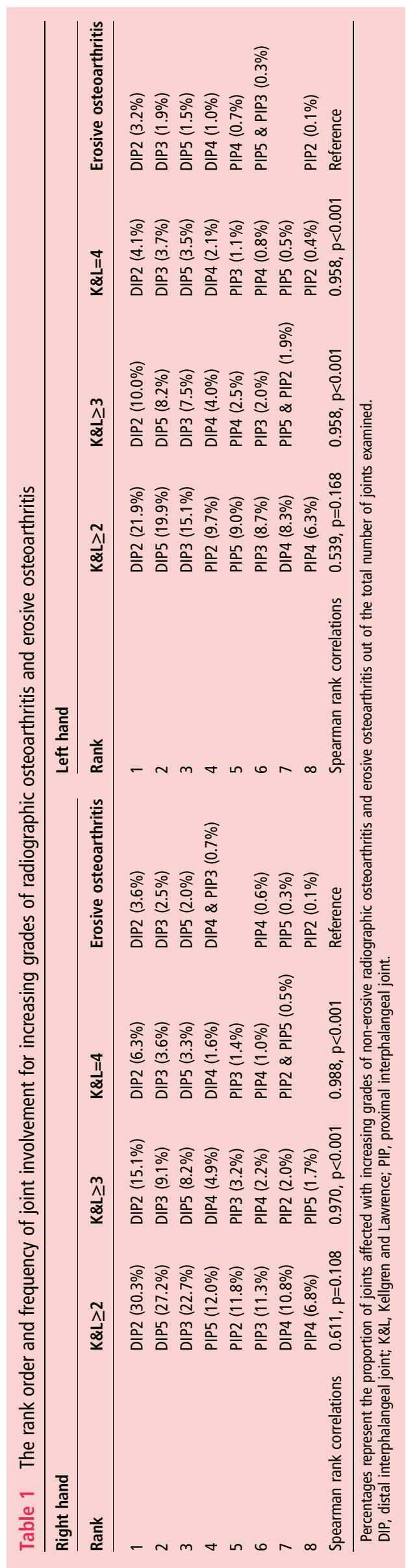


Table 2 Patterns of joint involvement by symmetry, row and ray for increasing grades of radiographic osteoarthritis and erosive osteoarthritis

\begin{tabular}{|c|c|c|c|c|c|c|c|c|}
\hline \multirow[b]{2}{*}{ Groups of Joints } & \multicolumn{2}{|c|}{$K \& L \geq 2$} & \multicolumn{2}{|c|}{$K \& L \geq 3$} & \multicolumn{2}{|c|}{$K \& L=4$} & \multicolumn{2}{|c|}{ Erosive osteoarthritis } \\
\hline & $\mathrm{aOR}$ & $(95 \% \mathrm{Cl})$ & $\mathrm{aOR}$ & $(95 \% \mathrm{Cl})$ & $\mathrm{aOR}$ & $(95 \% \mathrm{Cl})$ & $\mathrm{aOR}$ & $(95 \% \mathrm{Cl})$ \\
\hline Same joint, opposite hand & 3.8 & (3.1 to 4.5 ) & 6.0 & (4.2 to 8.5$)$ & 6.0 & (3.4 to 10.5 ) & 6.5 & (3.0 to 14.1 ) \\
\hline Same row, same hand & 3.2 & (2.7 to 3.8 ) & 3.1 & (2.1 to 4.6 ) & 4.2 & (2.3 to 7.8 ) & 2.0 & (0.8 to 5.0$)$ \\
\hline Same ray, same hand & 0.9 & (0.7 to 1.2$)$ & 0.5 & (0.3 to 0.9 ) & 0.3 & (0.1 to 0.8 ) & 0.3 & (0.03 to 2.5$)$ \\
\hline
\end{tabular}

aOR, adjusted OR for age, gender, hand joint, hand side and number of affected interphalangeal joints; K\&L, Kellgren and Lawrence.

\section{DISCUSSION}

Our analysis has identified an association between selected metabolic factors, notably dyslipidaemia, and the risk of erosive hand osteoarthritis in a population sample of symptomatic individuals. Reference groups were individuals with moderate-severe and severe non-erosive hand osteoarthritis and were selected from the same underlying population. This is important given the similar pattern of joint involvement and clustering by symmetry, row and ray among individuals with erosive osteoarthritis and those with increasing grades of non-erosive hand osteoarthritis. Our findings, if confirmed in other longitudinal studies, may suggest a role for systemic metabolic factors in the evolution from severe hand osteoarthritis to erosive osteoarthritis.

This research directly compared the frequency and patterning of joint involvement in erosive osteoarthritis to joints with increasing grades of radiographic osteoarthritis and found strong similarities between erosive osteoarthritis and moderatesevere and severe non-erosive osteoarthritis. Our study confirms that erosive osteoarthritis, like more severe hand osteoarthritis, targets the DIP joints more frequently than the PIP joints, and clustering of joint involvement was strongest for symmetry, followed by row and then ray. ${ }^{3} 12141626$ The same similarity of patterning was less evident for less severe grades of osteoarthritis that required only the presence of a definite osteophyte. This might imply that erosive osteoarthritis is less strongly related to osteophytosis than joint space narrowing, which is in keeping with research by Bijsterbosch et al, ${ }^{27}$ who found that erosive evolution in a joint was associated with the presence of joint space narrowing at baseline but not osteophytes. Together, these observations support the consecutive anatomical phases of the Verbruggen-Veys's scoring system. ${ }^{6}$

We examined a number of metabolic risk factors that have been associated with osteoarthritis and hand osteoarthritis. ${ }^{28-31}$ We found that compared with moderate-severe and severe non-erosive hand osteoarthritis, individuals with erosive osteoarthritis were more likely to have a diagnosis and consultation for dyslipidaemia and be classed with metabolic syndrome. Erosive evolution has been found to cluster strongly within

Table 3 Demographic and radiographic characteristics of individuals with erosive osteoarthritis compared to the reference groups with increasing grades of non-erosive radiographic hand osteoarthritis

\begin{tabular}{|c|c|c|c|}
\hline & $K \& L \geq 3$ & $K \& L=4$ & Erosive osteoarthritis \\
\hline Number & $\mathrm{n}=193$ & $n=68$ & $n=80$ \\
\hline Age range, years & $52-91$ & $55-85$ & $51-87$ \\
\hline Mean age, years (SD) & $68.9(8.1)$ & $69.1(7.5)$ & $69.2(7.8)$ \\
\hline Female gender & $79.3 \%$ & $79.4 \%$ & $83.8 \%$ \\
\hline Thumb IPJ osteoarthritis* & $63.7 \%$ & $69.1 \%$ & $68.8 \%$ \\
\hline Thumb MCP osteoarthritis* & $14.0 \%$ & $16.2 \%$ & $23.8 \%$ \\
\hline First CMC osteoarthritis* & $71.0 \%$ & $66.2 \%$ & $76.3 \%$ \\
\hline Trapezioscaphoid joint osteoarthritis* & $30.6 \%$ & $27.9 \%$ & $29.1 \%$ \\
\hline MCP osteoarthritis $\dagger$ & $30.6 \%$ & $35.3 \%$ & $32.5 \%$ \\
\hline Symptomatic radiographic knee osteoarthritis & $68.5 \%$ & $59.7 \%$ & $55.4 \%$ \\
\hline Manual occupation & $56.3 \%$ & $56.5 \%$ & $48.6 \%$ \\
\hline Attended higher education & $14.9 \%$ & $16.7 \%$ & $21.5 \%$ \\
\hline Family history of arthritis & $68.4 \%$ & $63.2 \%$ & $71.2 \%$ \\
\hline Previous hand injury & $25.4 \%$ & $24.6 \%$ & $32.9 \%$ \\
\hline Excessive hand use at work or in pastimes & $77.6 \%$ & $76.2 \%$ & $83.8 \%$ \\
\hline $\mathrm{BMl}>30 \mathrm{~kg} / \mathrm{m}^{2}$ & $34.4 \%$ & $35.3 \%$ & $37.5 \%$ \\
\hline Type 2 diabetes or impaired fasting glucose & $9.3 \%$ & $10.3 \%$ & $6.2 \%$ \\
\hline Hypertension & $37.8 \%$ & $29.4 \%$ & $43.8 \%$ \\
\hline Dyslipidaemia & $6.2 \%$ & $8.8 \%$ & $21.2 \%$ \\
\hline Prescribed a lipid-regulating drug & $17.1 \%$ & $16.2 \%$ & $20.0 \%$ \\
\hline Metabolic syndrome§ & $4.1 \%$ & $2.9 \%$ & $11.2 \%$ \\
\hline
\end{tabular}


Table 4 Risk factors for erosive osteoarthritis compared to reference groups with non-erosive moderate-severe and severe radiographic hand osteoarthritis

\begin{tabular}{|c|c|c|}
\hline Exposure & $\begin{array}{l}\text { Erosive osteoarthritis } \\
\text { vs } K \& L \geq 3 \\
\text { aOR }(95 \% \text { Cl) }\end{array}$ & $\begin{array}{l}\text { Erosive osteoarthritis } \\
\text { vs } K \& L=4 \\
\text { aOR }(95 \% \mathrm{Cl})\end{array}$ \\
\hline Female gender & $1.4(0.7$ to 2.7$)$ & $1.3(0.6$ to 3.1$)$ \\
\hline Manual occupation & $0.7(0.4$ to 1.2$)$ & 0.7 (0.4 to 1.4$)$ \\
\hline Attended higher education & 1.6 (0.8 to 3.1$)$ & $1.3(0.6$ to 3.0$)$ \\
\hline Family history of arthritis & $1.2(0.7$ to 2.1$)$ & $1.4(0.7$ to 2.9$)$ \\
\hline Previous hand injury & $1.5(0.8$ to 2.7$)$ & $1.5(0.7$ to 3.3$)$ \\
\hline $\begin{array}{l}\text { Excessive hand use at work } \\
\text { or in pastimes }\end{array}$ & $1.6(0.8$ to 3.2$)$ & $1.7(0.8$ to 4.0$)$ \\
\hline $\mathrm{BMI}>30 \mathrm{~kg} / \mathrm{m}^{2}$ & 1.1 (0.6 to 1.9$)$ & $1.1(0.5$ to 2.1$)$ \\
\hline $\begin{array}{l}\text { Type } 2 \text { diabetes or } \\
\text { impaired fasting glucose }\end{array}$ & $0.6(0.2$ to 1.8$)$ & 0.5 (0.2 to 1.8$)$ \\
\hline Hypertension & $1.2(0.7$ to 2.1$)$ & 1.8 (0.9 to 3.5$)$ \\
\hline Dyslipidaemia & 4.7 (2.1 to 10.6$)$ & 3.4 (1.2 to 9.6$)$ \\
\hline $\begin{array}{l}\text { Prescribed a } \\
\text { lipid-regulating drug }\end{array}$ & 1.2 (0.6 to 2.4$)$ & $1.4(0.6$ to 3.2$)$ \\
\hline Metabolic syndrome* & 2.7 (1.0 to 7.1$)$ & $3.2(0.8$ to 13.2$)$ \\
\hline \multicolumn{3}{|c|}{$\begin{array}{l}\text { aOR, adjusted OR for age and gender; BMI, body mass index; K\&L, Kellgren and } \\
\text { Lawrence. } \\
{ }^{*} \text { Metabolic syndrome was defined as the presence of three or more of the following: } \\
\text { BMI }>30 \mathrm{~kg} / \mathrm{m}^{2} \text {, hypertension, dyslipidaemia and type } 2 \text { diabetes or impaired fasting } \\
\text { glucose. }\end{array}$} \\
\hline
\end{tabular}

individuals, which suggests that the evolution of hand osteoarthritis to erosive osteoarthritis has a large systemic component. ${ }^{27}$ A previous study found BMI to be increased in individuals with erosive osteoarthritis compared to non-erosive osteoarthritis. ${ }^{8}$ This was not replicated in the present findings; however, the current analysis was designed to compare risk factors for erosive osteoarthritis to more severe hand osteoarthritis to determine whether they have the same risk factor profile. Therefore, BMI might be a risk factor for more severe hand osteoarthritis, but it does not discriminate between those with severe hand osteoarthritis and erosive osteoarthritis. Another study found significantly increased odds of hypercholesterolaemia in patients with erosive and non-erosive osteoarthritis compared to controls, but no assessment was made between erosive osteoarthritis and more severe non-erosive hand osteoarthritis. ${ }^{32}$ Increased serum cholesterol has been shown to be a risk factor for generalised osteoarthritis, ${ }^{33-35}$ and the use of statins has been found to lead to a reduction in both the incidence and progression of osteoarthritis, ${ }^{36} 37$ supporting the lack of association seen in the current analyses with lipid-regulating drugs. The exact mechanism is not yet known but osteoarthritis is believed to share similar biochemical and inflammatory pathways to metabolic disorders, ${ }^{38} 39$ and dyslipidaemia may alter lipid metabolism in a number of joint tissues. ${ }^{40-42}$ The association between osteoarthritis and a number of metabolic risk factors has been noted in a number of studies. ${ }^{29} 3043$ A population-based study recently demonstrated a significant dose-response relationship between the presence, incidence and progression of knee osteoarthritis and increasing numbers of metabolic factors. ${ }^{31}{ }^{44}$ Therefore, as we found in this analysis, more severe forms of osteoarthritis, which include erosive osteoarthritis, may be associated with the presence of multiple metabolic factors.

Erosive osteoarthritis has been considered a separate disease entity as unlike non-erosive hand osteoarthritis it typically has an abrupt onset. ${ }^{2}{ }^{3}$ Synovial pathology has shown an intense hypertrophy with acute inflammatory changes consistent with inflammatory arthritis. ${ }^{14}$ In one study, $15 \%$ of erosive osteoarthritis patients later developed seropositive rheumatoid arthritis. ${ }^{45}$ However, once the inflammatory phase has diminished histological samples are comparable to non-erosive hand osteoarthritis, suggesting inflammation is only a transitory phase. ${ }^{46}$ A further indication that erosive osteoarthritis may be a more severe form of hand osteoarthritis is that erosive osteoarthritis is seen in association with the same radiographic features of osteoarthritis and never in the absence of osteoarthritic changes. ${ }^{2-4}$ Research, including the present findings, has found that erosive osteoarthritis frequently affects the same hand joints as more severe non-erosive hand osteoarthritis. ${ }^{14}$ Nodes often occur in individuals with erosive osteoarthritis, ${ }^{47}$ and the greater clinical burden in erosive osteoarthritis compared to non-erosive osteoarthritis is thought to be largely due to the increased presence of nodes. ${ }^{11}$ Levels of serum hyaluronic acid, an important component of articular cartilage and synovial fluid, are increased in individuals with erosive compared to non-erosive osteoarthritis, signifying greater disease activity. ${ }^{10}$ However, the potential erosive-specific risk factors of dyslipidaemia and metabolic syndrome found in the current research may indicate why some go on to develop a more severe form of hand osteoarthritis.

A few methodological limitations should be considered when interpreting the findings of this study. Although all individuals included in the current analysis had recent hand symptoms, those from CASK were recruited on the basis on having knee pain and could have a more widespread form of osteoarthritis; however, no excess in the frequency of erosive or non-erosive hand osteoarthritis was seen in the CASK participants. This study was limited by its small sample size as erosive osteoarthritis is a relatively rare condition; nevertheless, other population-based studies, such as Framingham and Rotterdam, had comparable numbers with erosive osteoarthritis. ${ }^{8} 15$ The focus of this research was specifically on the radiographic presentation of erosive osteoarthritis in relation to increasing grades of non-erosive hand osteoarthritis and not the clinical or symptomatic aspects of the condition. General practice medical records were used to determine the metabolic exposures, except for BMI. However, all doctors making a diagnosis of a metabolic condition would be working to UK clinical guidelines. Furthermore, we considered whether individuals with erosive osteoarthritis might be more likely visit their general practitioner thereby increasing the possibility of the diagnosis of a metabolic risk factor. Sensitivity analysis found this was not the case. Finally, in this analysis individuals with prevalent erosive osteoarthritis were used along with a limited retrospective period for the ascertainment of metabolic exposures. A longer period of retrospective review and the use of incident erosive osteoarthritis would allow a more thorough analysis to be completed.

In conclusion, the frequency and patterning of joint involvement in erosive osteoarthritis is similar to that seen in moderate-severe and severe non-erosive radiographic osteoarthritis, which is consistent with erosive osteoarthritis being a more severe form of hand osteoarthritis rather than a distinct entity. Metabolic exposure, and dyslipidaemia in particular, may be associated with erosive osteoarthritis.

Acknowledgements The authors would like to acknowledge the contributions of Professor Peter Croft, Professor Elaine Hay, Dr Laurence Wood, Dr Elaine Thomas, June Handy, Charlotte Purcell, Catherine Tyson, Professor Chris Buckland-Wright and Professor lain McCall for aspects of the conception and design of the study and the acquisition of data. Dr Jacqueline Saklatvala, Carole Jackson, Julia Maheson, Janet Wisher, Sandra Yates, Krystina Wallbank and Jean Bamford from the 
department of radiography, Haywood Hospital, have contributed specifically to the acquisition of radiographs. They also wish to acknowledge June Handy for grading the CASK study radiographs for osteoarthritis. The authors would also like to thank the administrative and health informatics staff at the Arthritis Research UK Primary Care Centre at Keele University, as well as the staff and patients of the participating general practices.

Contributors The authors contributed to the manuscript as follows: Conception and design: MM, EN, WYK, GP, MK, DvdW, HM, KD. Data acquisition: MM, EN, WYK, GP, HM, KD. Analysis and interpretation of the data: MM, EN, WYK, GP, MK, DvdW. Drafting of the article: MM, EN, WYK, GP, MK, DvdW, HM, KD. Final approval of the article: MM, EN, WYK, GP, MK, DvdW, HM, KD.

Funding This work was supported by programme grants awarded by the Medica Research Council, UK (grant code: G9900220) and Arthritis Research UK (grant code: 18174), and NHS service support costs were provided by Support for Sciences funding secured from North Staffordshire Primary Care Consortium The funders did not contribute to data collection, analysis or interpretation of the data, manuscript preparation or submission.

\section{Competing interests None.}

Ethics approval The North Staffordshire Local Research Ethics Committee approved both the CASHA and CASK studies (LREC project no. 1430).

Provenance and peer review Not commissioned; externally peer reviewed.

Open Access This is an Open Access article distributed in accordance with the Creative Commons Attribution Non Commercial (CC BY-NC 3.0) license, which permits others to distribute, remix, adapt, build upon this work non-commercially, and license their derivative works on different terms, provided the original work is properly cited and the use is non-commercial. See: http://creativecommons.org/ licenses/by-nc/3.0

\section{REFERENCES}

1 Crain DC. Interphalangeal osteoarthritis. JAMA 1961;175:1049-53.

2 Peter JB, Pearson CM, Marmor L. Erosive osteoarthritis of the hands. Arthritis Rheum 1966;9:365-88

3 Ehrlich GE. Inflammatory osteoarthritis-l. The clinical syndrome. J Chron Dis 1972;25:317-28

4 Punzi L, Ramonda R, Sfriso P. Erosive osteoarthritis. Best Pract Res Clin Rheumatol 2004;18:739-58.

5 Belhorn LR, Hess EV. Erosive osteoarthritis. Semin Arthritis Rheum 1993:22:298-306.

6 Verbruggen G, Veys EM. Numerical scoring systems for the anatomic evolution of osteoarthritis of the finger joints. Arthritis Rheum 1996;39:308-20.

7 Verbruggen G, Wittoek R, Vander Cruyssen B, et al. Morbid anatomy of 'erosive osteoarthritis' of the interphalangeal finger joints: an optimised scoring system to monitor disease progression in affected joints. Ann Rheum Dis 2010;69:862-7.

8 Kwok WY, Kloppenburg M, Rosendaal FR, et al. Erosive hand osteoarthritis: its prevalence and clinical impact in the general population and symptomatic hand osteoarthritis. Ann Rheum Dis 2011;70:1238-42.

9 Pattrick $M$, Aldridge $S$, Hamilton $E$, et al. A controlled study of hand function in nodal and erosive osteoarthritis. Ann Rheum Dis 1989:48:978-82.

10 Filkova M, Senolt L, Braun M, et al. Serum hyaluronic acid as a potential marker with predictive value for further radiographic progression of hand osteoarthritis. Osteoarthritis Cartilage 2009:17:1615-19.

11 Bijsterbosch J, Watt I, Meulenbelt I, et al. Clinical burden of erosive hand osteoarthritis and its relationship to nodes. Ann Rheum Dis 2010:69:1784-8.

12 Addimanda O, Mancarella L, Dolzani $\mathrm{P}$, et al. Clinical and radiographic distribution of structural damage in erosive and nonerosive hand osteoarthritis. Arthritis Care Res 2012;64:1046-53.

13 Zhang W, Doherty M, Leeb BF, et al. EULAR evidence-based recommendations for the diagnosis of hand osteoarthritis: report of a task force of ESCISIT. Ann Rheum Dis 2009:68:8-17.

14 Cobby M, Cushnaghan J, Creamer $\mathrm{P}$, et al. Erosive osteoarthritis: is it a separate disease entity? Clin Radiol 1990;42:258-63.

15 Haugen IK, Englund M, Aliabadi $P$, et al. Prevalence, incidence and progression of hand osteoarthritis in the general population: the Framingham Osteoarthritis Study. Ann Rheum Dis 2011;70:1581-6.

16 Egger P, Cooper C, Hart DJ, et al. Patterns of joint involvement in osteoarthritis of the hand: the Chingford Study. J Rheumatol 1995:22:1509-13.

17 Dahaghin S, Bierma-Zeinstra SM, Ginai AZ, et al. Prevalence and pattern of radiographic hand osteoarthritis and association with pain and disability (the Rotterdam Study). Ann Rheum Dis 2005;64:682-7.

18 Kloppenburg M, Kwok WY. Hand osteoarthritis-a heterogeneous disorder. Nat Rev Rheumatol 2011:8:22-31.

19 Myers $\mathrm{H}$, Nicholls $\mathrm{E}$, Handy J, et al. The clinical assessment study of the hand (CAS-HA): a prospective study of musculoskeletal hand problems in the genera population. BMC Musculoskelet Disord 2007;8:85.
20 Bowling A. Research methods in health: investigating health and health sciences, 3rd edn. Maidenhead: Open University Press, 2009: 194-6.

21 Peat G, Thomas E, Handy J, et al. The knee clinical assessment study-CAS(K). A prospective study of knee pain and knee osteoarthritis in the general population. BMC Musculoskelet Disord 2004;5:4.

22 Lawrence JS. Osteo-arthrosis. In: Lawrence JS. ed. Rheumatism in populations. London: William Heinemann Medical Books, 1977: 98-155.

23 Porcheret $M$, Hughes $\mathrm{R}$, Evans $\mathrm{D}$, et al. Data quality of general practice electronic health records: the impact of a program of assessments, feedback, and training. J Am Med Inform Assoc 2004;11:78-86.

24 Jordan K, Clarke AM, Symmons DP, et al. Measuring disease prevalence: a comparison of musculoskeletal disease using four general practice consultation databases. Br J Gen Pract 2007:57:7-14.

25 Peat G, Thomas E, Handy J, et al. The knee clinical assessment study-CAS(K). A prospective study of knee pain and knee osteoarthritis in the general population: baseline recruitment and retention at 18 months. BMC Musculoskelet Disord 2006;7:30.

26 Smith D, Braustein EM, Brandt KD, et al. A radiographic comparison of erosive osteoarthritis and idiopathic nodal osteoarthritis. I Rheumatol 1992:19:898-904.

27 Bijsterbosch J, van Bemmel JM, Watt I, et al. Systemic and local factors are involved in the evolution of erosions in hand osteoarthritis. Ann Rheum Dis 2011;70:326-30.

28 Bagge E, Bjelle A, Edén $\mathrm{S}$, et al. Factors associated with radiographic osteoarthritis: results from the population study of 70-year-old people in Göteborg. I Rheumatol 1991;18:1218-22

29 Dahaghin S, Bierma-Zeinstra SM, Koes BW, et al. Do metabolic factors add to the effect of overweight on hand osteoarthritis? The Rotterdam Study. Ann Rheum Dis 2007;66:916-20.

30 Puenpatom RA, Victor TW. Increased prevalence of metabolic syndrome in individuals with osteoarthritis: an analysis of NHANES III data. Postgrad Med 2009:121:9-20.

31 Yoshimura N, Muraki S, Oka H, et al. Association of knee osteoarthritis with the accumulation of metabolic risk factors such as overweight, hypertension, dyslipidemia, and impaired glucose tolerance in Japanese men and women: the ROAD Study. J Rheumatol 2011;38:921-30.

32 Addimanda 0 , Mancarella L, Dolzani $\mathrm{P}$, et al. Clinical associations in patients with hand osteoarthritis. Scand J Rheumatol 2012:41:310-13.

33 Hart DJ, Spector TD. The relationship of obesity, fat distribution and osteoarthritis in women in the general population: the Chingford Study. J Rheumatol 1993:20:331-5

34 Sturmer T, Sun Y, Sauerland $S$, et al. Serum cholesterol and osteoarthritis. The baseline examination of the UIm Osteoarthritis Study. J Rheumatol 1998:25:1827-32

35 Al-Arfaj AS. Radiographic osteoarthritis and serum cholesterol. Saudi Med 2003;24:745-7

36 Clockaerts S, Van Osch GJ, Bastiaansen-Jenniskens YM, et al. Statin use is associated with reduced incidence and progression of knee osteoarthritis in the Rotterdam Study. Ann Rheum Dis 2012:71:642-7.

37 Kadam UT, Blagojevic M, Belcher J. Statin use and clinical osteoarthritis in the general population: a longitudinal study. J Gen Intern Med 2013;28:943-9.

38 Katz JD, Agrawal S, Velasquez M. Getting to the heart of the matter: osteoarthritis takes its place as part of the metabolic syndrome. Curr Opin Rheumatol 2010:22:512-19.

39 Kadam UT, Holmberg A, Blagojevic M, et al. Risk factors for cardiovascular disease and future osteoarthritis-related arthroplasty: a population-based cohort study in men and women form Malmo, Sweden. Scand J Rheumatol 2011:40:478-85.

40 Aspden RM, Scheven BA, Hutchison JD. Osteoarthritis as a systemic disorder including stromal cell differentiation and lipid metabolism. Lancet 2001:357:1118-20.

41 Gkretsi V, Simopoulou T, Tsezou A. Lipid metabolism and osteoarthritis: lessons from atherosclerosis. Proq Lipid Res 2011;50:133-40.

42 Zhuo Q, Yang W, Chen J, et al. Metabolic syndrome meets osteoarthritis. Nat Rev Rheumatol 2012;8:729-37.

43 Sowers M, Karvonen-Gutierrez CA, Palmieri-Smith R, et al. Knee osteoarthritis in obese women with cardiometabolic clustering. Arthritis Rheum 2009;61:1328-36

44 Yoshimura N, Muraki S, Oka H, et al. Accumulation of metabolic risk factors such as overweight, hypertension, dyslipidaemia, and impaired glucose tolerance raises the risk of occurrence and progression of knee osteoarthritis: a 3-year follow-up of the ROAD Study. Osteoarthritis Cartilage 2012;20:1217-26.

45 Ehrlich GE. Inflammatory osteoarthritis-II The superimposition of rheumatoid arthritis. J Chron Dis 1972;252:635-43.

46 Greenway G, Resnick D, Weisman M, et al. Carpal involvement in inflammatory (erosive) osteoarthritis. J Can Assoc Radiol 1979;30:95-8.

47 Marshall M, Peat G, Nicholls E, et al. Subsets of symptomatic hand osteoarthritis in community-dwelling older adults in the United Kingdom: prevalence, interrelationships, risk factor profiles and clinical characteristics at baseline and 3-years. Osteoarthritis Cartilage. [Epub ahead of print 13 Aug 2013] doi:pii: S1063-4584 (13)00907-2. 10.1016/j.joca.2013.08.004 